\title{
Herramienta de Realidad Aumentada para Facilitar la Enseñanza en Contextos Educativos Mediante el Uso de las TICs
}

\author{
Jorge Ierache, Santiago Igarza, Nahuel A. Mangiarua, Martín E. Becerra, Sebastián A. Bevacqua, \\ Nicolás N. Verdicchio, Fernando M. Ortiz, Diego R. Sanz, Nicolás D. Duarte, Matías Sena \\ Departamento de Ingeniería e Investigaciones Tecnológicas \\ Universidad Nacional de La Matanza \\ San Justo, Buenos Aires \\ jierache@ing.unlam.edu.ar, realidadaumentada.unlam@gmail.com
}

\begin{abstract}
Resumen-La Realidad Aumentada agrega elementos virtuales al entorno Real, proporcionándonos información de interés para el usuario aprovechando la infraestructura de las TICs. De esta manera, el entorno real es enriquecido con información mejorando así las experiencias en diferentes áreas, tales como entretenimiento, salud, Industria y principalmente los entornos educativos. En este artículo se presenta un framework que facilite a los educadores la enseñanza de los contenidos y aumente la participación de los alumnos, usando un juego de mesa. De esta manera, el alumnado interactúa directamente con los contenidos virtuales con el fin de afianzar sus conocimientos en diferentes áreas.
\end{abstract}

Palabras clave - Realidad Aumentada, dispositivos móviles aplicados en RA, aplicación de RA en la educación, Framework de RA.

\section{INTRODUCCIÓN}

En la actualidad, los avances tecnológicos han permitido que la experiencia de Realidad Aumentada sea posible tanto en computadoras personales como en Smartphones. Estos últimos son los que ofrecen mayor usabilidad de las aplicaciones creadas con esta tecnología al ser dispositivos potentes, portables y versátiles debido a los servicios y sensores que brindan.

Bajo el término de realidad aumentada [1] (en inglés Augmented Reality o AR) se agrupan aquellas tecnologías que permiten la superposición, en tiempo real, de imágenes o marcadores $^{1} \mathrm{o}$ información generados virtualmente, sobre imágenes del mundo real. De esta manera, se crea un entorno en el que la información y los objetos virtuales se fusionan con los objetos reales, ofreciendo una experiencia tal para el usuario, que puede llegar a pensar que forma parte de su realidad cotidiana. La Realidad Aumentada es una tecnología que ayuda a enriquecer nuestra percepción de la realidad con una nueva lente gracias a la cual la información del mundo real se complementa con la del digital.

La Realidad Aumentada (RA) [2] agrega información sintética a un ambiente real. La diferencia principal entre esta y la Realidad Virtual (RV) es que la última implica inmersión del participante en un mundo totalmente virtual; en cambio RA implica mantenerse en el mundo real con agregados virtuales.

${ }^{1}$ Marcador, es una imagen impresa que proporciona una referencia espacial, permitiendo al dispositivo imprimir la información virtual en el entorno real captado relativo al mismo.

Ierache, J., Igarza, S., Mangiarua, N., Becerra, M., Bevacqua, S., Verdicchio, N., Ortiz, F., Sanz, D., Duarte, N., Sena, M., 2014. Herramienta de Realidad Aumentada para facilitar la enseñanza en contextos educativos mediante el uso de las TICs.

Revista Latinoamericana de Ingeniería de Software, 2(6): 365-368, ISSN 2314-2642
Para Ronald Azuma [3, 4], un sistema de RA es aquél que cumple con tres condiciones de base: (a) Combina la realidad y lo virtual. Al mundo real se le agregan objetos sintéticos que pueden ser visuales como texto $\mathrm{u}$ objetos $3 \mathrm{D}$ (wireframe o fotorealistas), auditivos, sensibles al tacto y/o al olfato, (b) Es interactivo en tiempo real. El usuario ve una escena real con objetos sintéticos agregados, que le que ayudarán a interactuar con su contexto, (c) Las imágenes son registradas en espacios 3D. La información virtual tiene que estar vinculada espacialmente al mundo real de manera coherente. Se necesita saber en todo momento la posición del usuario respecto al mundo real y de esta manera puede lograrse el registro de la mezcla entre información real y sintética. En síntesis un sistema de RA tiene tres requerimientos según Ronald Azuma: combina la realidad con información sintética, los objetos virtuales están registrados en el mundo real, es interactivo en tiempo real.

En base a lo anterior descripto, el presente trabajo se basa en la generación de una herramienta en el contexto de Realidad Aumentada para contribuir al aprendizaje de contenidos educativos, con la aplicación de los ultimas avances en las TIC's de manera de crear una experiencia donde los alumnos interactúen con dichos contenidos. La propuesta del Grupo de Realidad Aumentada es proporcionar a los Educadores, un framework para dispositivos móviles que permita enriquecer la experiencia de un juego de mesa enfocado a un contexto educativo en el que se formulan preguntas previamente desarrolladas en los contenidos. Por ejemplo, preguntas de interés general, historia argentina,

\section{ESTADO DEL ARTE}

\section{A. Realidad Aumentada en ámbitos educativos}

En el estado del arte enfocado a los ámbitos educativos, podemos encontrar diferentes proyectos que enriquecen los métodos de enseñanza.

En [5] se describe una herramienta RA llamada AuthorAR. Esta herramienta de autor está orientada a la creación de actividades educativas de RA, con la inclusión de plantillas específicas para el escenario de educación especial, con foco en el entrenamiento de competencias comunicacionales. Las dos plantillas que desarrollaron hasta el momento de su publicación son: una para actividades de exploración y otra para actividades de estructuración de frases. salud, biología, etc. 
Por un lado se desarrollaron herramientas para facilitarles a personas con diferentes dificultades, la enseñanza de contenidos educativos. Un ejemplo es PictogramRoom [6], es un proyecto que involucra una habitación de Realidad Aumentada para enseñar a comprender los pictogramas que permiten la comunicación a personas con trastornos del espectro del autismo. En otro orden Eyering [7], plantea un anillo de Realidad Aumentada equipado con una pequeña cámara, un procesador, conectividad Bluetooth y retroalimentación auditiva, a través de un dispositivo portátil, que podría ayudar a las personas con dificultades visuales a identificar objetos y leer texto. E-labora [8], incorpora la Realidad Aumentada y la tecnología $3 \mathrm{G}$ en actividades de entrenamiento y formación profesional. Este proyecto pretende mejorar la integración de las personas con discapacidad intelectual en el lugar de trabajo. En materia de juegos se han obtenido diversos resultados aplicando RA, como por ejemplo Virtuoso [9], un Juego educativo multiusuario que corre en el interior de un museo de Historia y Arte. El objetivo es ubicar cronológicamente, de izquierda a derecha, las palabras claves en las ranuras de la línea de tiempo, representada en la pared mediante marcadores. Usando la tecnología RA, cada ítem es representado virtualmente en la línea de tiempo y proporciona información de base acerca del mismo, con su nombre y una descripción sintética.

\section{HERRAMIENTA DE REALIDAD AUMENTADA}

La herramienta propuesta consiste en un framework que permite articular con el plano de un juego que contiene casilleros y se encuentra desplegado en un tablero físico, los contenidos de Realidad Aumentada previamente configurados, seleccionados y almacenados en función de la temática. La aplicación ejecuta videos sobre el casillero de la superficie del tablero, proporcionando la sensación de que el video se posiciona y ejecuta realmente en el casillero físico, generando de esta forma una aumentación de la realidad. Cada video ofrece tres opciones de las cuales el jugador elegirá una mediante un toque en la pantalla de su dispositivo.

El proceso consiste en que cada alumno participante del juego toca el dado virtual para que su ficha virtual avance a través de los casilleros del tablero físico. En cada casillero aparecerá un video aleatorio correspondiente a la temática seleccionada, sobre el que el sistema le hará una pregunta, para que este pueda seguir avanzando. En caso que conteste incorrectamente, perderá el turno y seguirá otro alumno. En caso de acertar, el alumno sigue jugando. El alumno al llegar a la meta no solamente alcanza el objetivo de completar el recorrido del juego, sino que se obtiene una puntuación del mismo y detalles de sus aciertos y errores, sobre los cuales se puede recurrir para luego fijar los contenidos que el alumno no pudo superar en el juego.

\section{A. Framework}

El desarrollo se realizó en Android y se empleó las herramientas Unity3D [10] y Vuforia [11].

A continuación se explicara el funcionamiento del framework. En la Fig. 1 se puede observar, los componentes del framework.

La aplicación está compuesta por el controlador del juego que administra el funcionamiento general, delegando tareas a los componentes restantes durante la ejecución del mismo. Otro componente es Contenidos Target, se encarga de seleccionar el contenido para cargar los videos y las opciones sobre los marcadores para el jugador de turno. En cuanto a la representación de los elementos virtuales sobre el tablero, el componente Elementos Juego se encarga de administrar el Tablero, Dado, Ficha del Jugador 1 y Ficha de Jugador 2. Cada una se encarga de representar cada elemento virtual respectivamente. Por último, el componente Controlador Respuesta se encarga de determinar el resultado según la opción que elijan los jugadores.

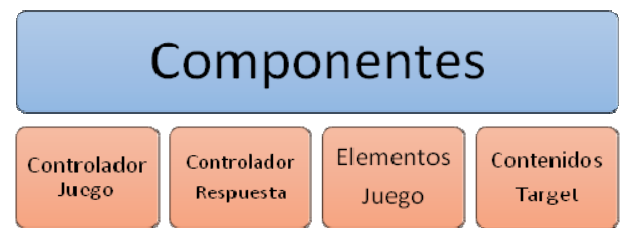

Fig. 1. Componentes del Framework

En la Fig. 2, se puede observar un diagrama de clases conceptuales que aporta una visión más detallada de las relaciones más relevantes entre entidades que conforman los componentes de la aplicación.

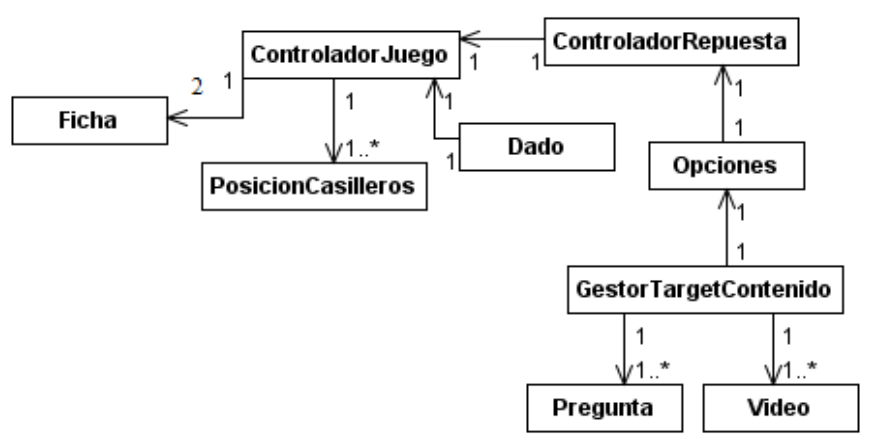

Fig. 2. Diagrama de clases

A continuación, se muestra una secuencia que explica la secuencia normal del framework. El escenario consiste en que el usuario toca el dado virtual de la aplicación para que el controlador del juego indique a la ficha su movimiento al casillero correspondiente y que le indique al gestor de contenido, proceda la carga del video con las opciones correspondientes.

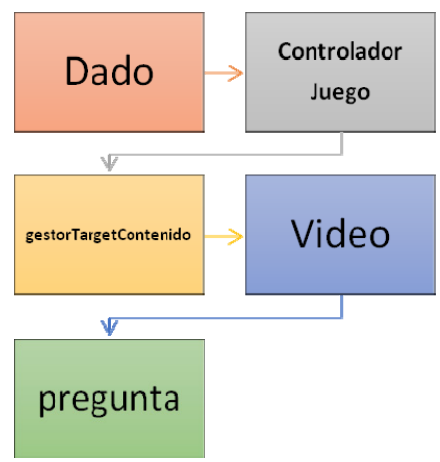

Fig. 3. Secuencia normal

\section{CAso de Estudio, Aplicación Juega Pulsar Play}

\section{A. Framework de aplicación}

Para utilizar el framework, los usuarios deben contar con un Smartphone que cuente con sistema operativo Android y la aplicación presentada previamente instalada. Para jugar los usuarios deben enfocar el tablero con su dispositivo para que los elementos virtuales puedan ser visualizados a través del visor del dispositivo. Al iniciar el turno, el alumno que participa, toca el dado virtual para determinar cuántos 
casilleros avanzar. Luego, la ficha asociada al alumno jugador de turno se desplazará hasta la posición que fue determinada por el número aleatorio obtenido al tirar el dado, aumentando la realidad sobre el tablero físico de juego. A continuación, un video se ejecutará para realizar una pregunta al alumno en el marco del juego. Al finalizar la reproducción de video, se mostrarán las opciones en la que se debe elegir una como respuesta. Como resultado se puede esperar que el jugador continúe con su turno, si contesto correctamente. Caso contrario, pierde el turno para cedérselo al otro alumno participante. La partida finaliza cuando algún participante llega a la meta. El usuario aumenta la realidad del tablero físico de juego a través de su dispositivo móvil el que corre la aplicación Juega PulsAR Play [12] seleccionando marcadores temáticos correspondiente a las categorías de las preguntas, que contiene los videos de contenidos con los que interactúa el usuario en un ambiente de Realidad Aumentada, se muestra una imagen en la Fig. 4.

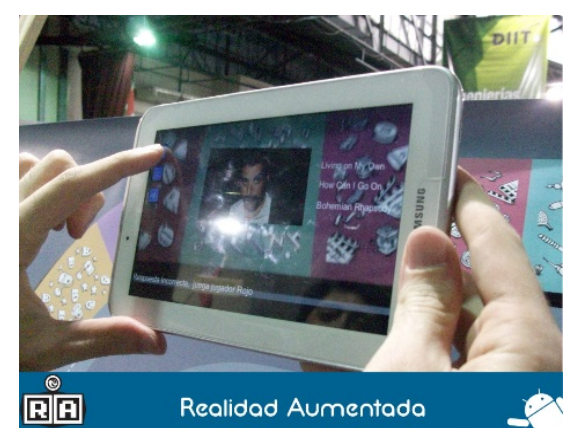

Fig. 4. Dispositivo móvil-usuario eligiendo una opción

Se describen a continuación marcadores utilizados en presentaciones iniciales del sistema desarrolladas durante las exposiciones: "Segunda Muestra Municipal de Ciencia y Tecnología - Plaza Ciencia 2013" y "ExpoProyecto UNLaM 2013" que tomaron lugar en el partido de La Matanza.

Se generaron contenidos y preguntas asociados a los marcadores, referido a diferentes temáticas, como: Actualidad Fig. 5a, Historia Fig. 5b, Música Fig. 5c, Cine Fig. 5d, Deporte Fig. 5e. Con estos contenidos se realizaron pruebas con el público visitante aplicando el framework desarrollado para la plataforma Android.

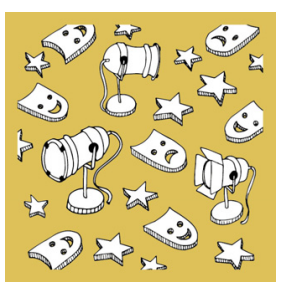

Fig. 5a. Actualidad

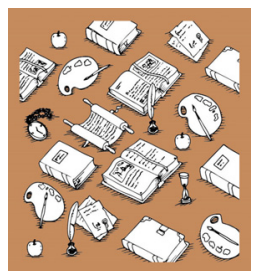

Fig. 5b. Historia

\section{B. Tablero físico}

Para utilizar el framework, en este caso se diseñó un tablero físico con el correspondiente plano del juego, como el que se observa en Fig. 6, en el que las fichas virtuales de los jugadores avanzan por un camino junto a los casilleros que representan las categorías temáticas identificadas en función de los marcadores, que presentan las preguntas, a partir de los videos en su dispositivo móvil. Cada casillero pertenece a una de las categorías.

Cada categoría tiene asociada videos de contenidos temáticos sobre los que realizan preguntas al alumno que debe seleccionar una opción luego de la reproducción de video.

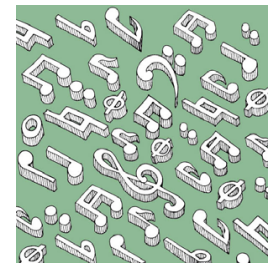

Fig. 5c. Música

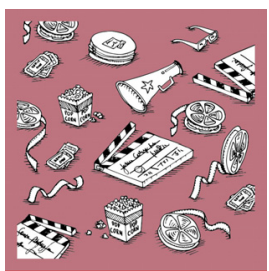

Fig. 5d. Películas

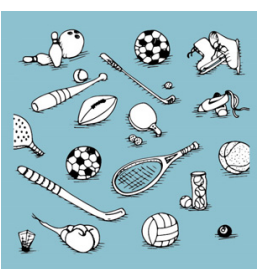

Fig. 5e. Deportes

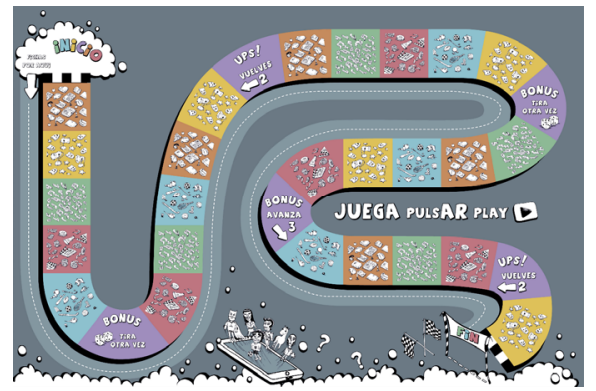

Fig. 6. Tablero físico

Las Fig. 5a hasta 5e funcionan como marcadores que indican una referencia espacial para el contenido virtual (videos y opciones para responder), los marcadores permiten que se adapte exitosamente el mundo real del tablero físico y actúan como identificadores de contenidos temáticos para el alumno que se encuentra participando del juego.

\section{Elementos virtuales}

La aplicación de RA, a través del empleo del Smartphone o Tablet, que es enfocada y posicionada en el tablero físico, presenta los elementos virtuales que aumenta la realidad, en este orden para su utilización la aplicación presenta: un dado virtual (Fig. 7a), las fichas virtuales para cada jugador (Fig. 7b), videos, respuestas posibles sobre las que el alumno tiene que elegir una opción (Fig. 8).
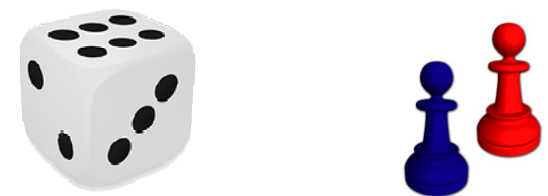

Fig. 7a Dado Virtual

Fig. $7 b$ Fichas de jugadores

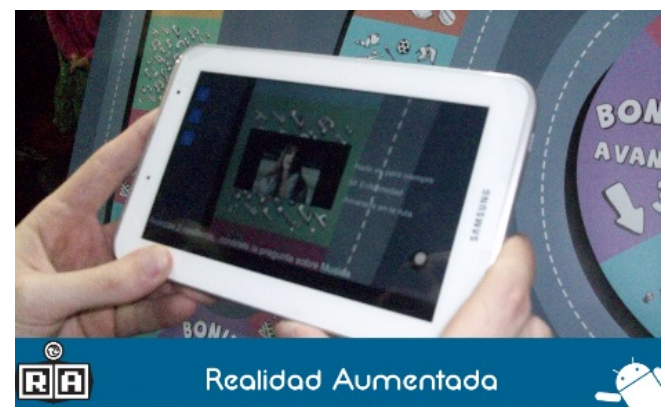

Fig. 8. Video con opciones para responder

\section{Modalidad de funcionamiento}

Para utilizar el framework, los usuarios deben contar con un Smartphone que cuente con sistema operativo Android con la aplicación previamente instalada. Para utilizarlo los usuarios deben enfocar el tablero con su dispositivo para que los elementos virtuales puedan ser visualizados a través del visor del dispositivo. Para iniciar el turno, el jugador correspondiente, toca el dado virtual para determinar cuántos casilleros avanzar. Luego, La ficha asociada al jugador de turno se desplazará hasta la posición que fue determinada por 
el número aleatorio obtenido. A continuación, un video se ejecutará para realizar una pregunta al jugador. Al finalizar la reproducción de video, se mostrarán las opciones en la que se debe elegir una como respuesta. Como resultado se puede esperar que el jugador continúe con su turno, si contesto correctamente. Caso contrario, pierde el turno para cederle el turno a su contrincante. La partida finaliza cuando un jugador llega a la meta.

Nuestro grupo de investigación realizó pruebas de este framework en la Expo Proyecto UNLaM 2013 a través de la aplicación Juega PulsAR Play, donde alumnos de escuelas secundarias y estudiantes universitarios de diversas carreras fueron invitados a participar de partidas de prueba. Durante los 3 días de la exposición se observaron resultados satisfactorios donde los alumnos demostraban gran interés por la mecánica del juego que recompensa el conocimiento y aprendizaje. Se observó también un alto nivel de incorporación de los contenidos expuestos en la instancia de prueba por parte de estudiantes que participaron de la prueba en más de una ocasión.

\section{CONCLUSIONES Y FUTURAS LÍNEAS DE TRABAJO}

La explotación del Framework en función del programa de estudios con el que cuentan los educadores para llevar a cabo la transmisión de conocimientos en un contexto educativo, requiere que se definan las categorías que se quieran abordar para segmentar las preguntas y respuestas posibles para que la aplicación funcione. Además se debe diseñar el tablero físico y los marcadores de acuerdo a las temáticas propuestas, ya que las categorías se usan como marcadores representativos de las mismas. Es posible también generar la imagen del tablero y los marcadores proyectándolos sobre el pizarrón o pared a fin de ahorrar costos y tiempos con la construcción física de los mismos. La colaboración de contenidos educativos para explotar en el Framework de RA, se puede realizar a través de un catálogo de contenidos de RA [13] el que actualmente se encuentra en desarrollo, permitiendo que se puedan publicar y compartir contenidos, marcadores, cuestionarios y resultados.

Debido a los resultados obtenidos en las experiencias previamente citadas, este tipo de tecnologías y en especial la herramienta presentada, contribuye a enriquecer los métodos de enseñanza. Nuestro framework hace uso de una nueva tecnología emergente como lo es la Realidad Aumentada que utiliza las TICs para añadir al entorno real de las personas, nuevas alternativas que enriquezcan los métodos de enseñanza de contenidos educativos. La línea de investigación a seguir es desarrollar nuevos incrementos del GestorTargetContenido para permitirle al usuario generar el contenido, marcadores y las preguntas para que el framework funcione a través del mismo facilitando la colaboración de contenidos de RA a través del catálogo de RA que se encuentra en desarrollo.

\section{AGRADECIMIENTOS}

Este trabajo es financiado en el marco del proyecto PROINCE C-168 del DIIT, nuestro agradecimiento a la Ing. Zaloa Urrutikoetxea quien dio origen a la conformación del grupo de RA de la UNLaM.

\section{REFERENCIAS}

[1] Fundación Telefónica, "Realidad aumentada: una nueva lente para ver el mundo", 1st ed., pp. 291-325, Jan. 2011.
[2] C. Manresa Yee, M. J. Abásolo, R. Más Sansó, M. Vénere, "Realidad virtual y realidad aumentada. Interfaces avanzadas", 1st ed., pp. 16-18, Universidad Nacional de La Plata, La Plata, Buenos Aires, Argentina, 2011.

[3] R. T. Azuma, "A survey of augmented reality", in Presence: Teleoperators and Virtual Environments, vol. 6, no. 4, pp. 355385, Aug. 1997.

[4] R. T. Azuma, Y. Baillot, R. Behringer, S. K. Feiner, S.J. Julier, B. MacIntyre, "Recent advances in augmented reality" in IEEE Computer Graphics and Applications, pp. 34-47, Nov-Dec, 2001.

[5] L. Moralejo, C. Sanz, P. Pesado, S. Baldassarri, "Avances en el diseño de una herramienta de autor para la creación de actividades educativas basadas en realidad aumentada" in XIX Congreso Argentino de Ciencias de la Computación - CACIC 2013, 1st ed., pp. 516-525, Jan. 2013.

[6] Pictogram room, http://www.pictogramas.org/proom/init.do? method=initTab, (Vigente: Noviembre 2014).

[7] Eyering, http://www.digitalavmagazine.com/2012/08/13/el-mitcrea-un-dispositivo-de-realidad-aumentada-para-ciegos-activado -por-voz/, (Vigente: Noviembre 2014).

[8] T. de Andrés, M. Satur Torre, "Augmented Reality for e-labora: aplicaciones móviles para trabajadores con discapacidad intelectual",http://www.qualcomm.com/media/documents/files/ wireless-reach-case-study-spain-augmented-reality-spanish-.pdf, (Vigente: Noviembre 2014).

[9] Á. Belcastro, "Realidad Aumentada. UNLP: Virtuoso", http://www.ing.unp.edu.ar/asignaturas/ias/realaum.pdf, (Vigente: Noviembre 2014)

[10] Unity3D, www.unity3d.com, (Vigente: Noviembre 2014).

[11] Vuforia, https://www.vuforia.com/, (Vigente: Noviembre 2014).

[12] Juega PulsAR Play, https://play.google.com/store/apps/details? $\mathrm{id}=$ com.unlam.realidadaumentada.juegapulsarplay, (Vigente: Noviembre 2014).

[13] J. Ierache, N. Mangiarua, S. A. Bevacqua, M. Becerra, N. Verdicchio, M. Sena, N. Duarte, D. Sanz, F. Ortiz, S. Igarza, "Sistema de catálogo para la asistencia a la creación, publicación, gestión y explotación de contenidos multimedia y aplicaciones de realidad aumentada" in XX Congreso Argentino de Ciencias de la Computación, 1st ed., art. 6627, Oct. 2014.

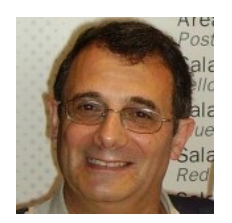

Jorge Ierache es Doctor en Ciencias Informáticas por la Facultad de Informática de la Universidad Nacional de la Plata, profesor adjunto de la Universidad Nacional de La Matanza y Director del Grupo de Realidad Aumentada Aplicada.

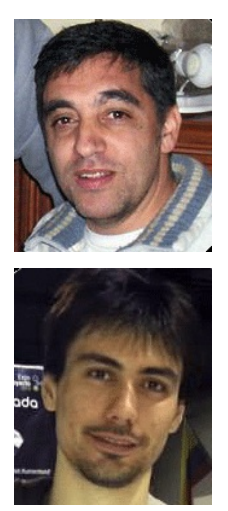

Santiago Igarza es Ingeniero en Informática, Director de Carrera de Ingeniería Informática de la Universidad Nacional de La Matanza, Codirector del Grupo de Realidad Aumentada Aplicada y egresado de la misma.

Nahuel Adiel Mangiarua es Ingeniero en Informática, ayudante de primera, miembro del Grupo de Realidad Aumentada Aplicada de la Universidad Nacional de La Matanza y egresado de la misma.

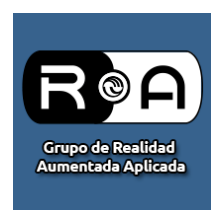

Martín Ezequiel Becerra, Sebastián Ariel Bevacqua, Nicolás Nazareno Verdicchio, Fernando Martín Ortiz, Diego Rubén Sanz, Nicolás Daniel Duarte y Matías Sena son estudiantes de la carrera de Ingeniería en Informática y becarios del Grupo de Realidad Aumentada Aplicada de la Universidad Nacional de La Matanza. 